\title{
A Fuzzy Preference Relation Based Method for Face Recognition by Gabor Filters
}

\author{
Soumak Biswas, Sripati Jha, Ramayan Singh \\ Department of Mathematics, National Institute of Technology, Jamshedpur, India. \\ Email: sbiswas.nitjsr@yahoo.com, sripati_jha_nitjsr@yahoo.co.in,rsingh.nitjsr@yahoo.com
}

\begin{abstract}
In this paper we have applied Gabor filter for fiducial point localization. After obtaining the fiducial points the number of fiducial points are reduced using a distance formula. The distance of each of this fiducial point is then calculated by the distance formula and stored in the database of the system. The same methodology is also applied on the input face which is to be matched with the faces available in the database. Then a fuzzy preference relation matrix is obtained . the largest eigen value of this matrix is then determined by algebraic method or numerical method depending on the order of the matrix. To apply the numerical method which is more easier for large order matrices we have used the $\mathrm{C}$ programming of this method. Once the largest eigen value is determined the corresponding priority vector can easily be obtained from which we can easily match the input face with the database.
\end{abstract}

Index terms - Fiducial pont, Priority vector, Largest Eigen value, Decision making (DM), Ranking.

\section{Introduction}

Facial recognition system is one of the most remarkable abilities human being enjoys. It is important for several aspect of our social life. Machine recognition of faces has recently emerged as one of the most important area amongst researchers in Mathematics and Computer science. As one of the most successful application of image analysis has recently got great deal of attention from researchers across the globe which is evident from the growing number of conferences and research that has taken places. Computer analysis of face images deals with visual signals (light reflected of the surface of a face) that is registered by a digital sensor or an array of pixel value.The pixel can encode intensity. Though a formal method of classifying faces was first proposed by Francis Galton in 1888. The use of face recognition is immense, face recognition technology has numerous law enforcement and commercial applications [7][16]. Face recognition can efficiently replace the use of pin numbers and passwords. The passwords and pin numbers are difficult to remember although they are very helpful for authentication but they can be easily stolen. Applications where face recognition can be used efficiently include secure transactions in e and $\mathrm{m}$ commerce. On the other hand Fuzzy preference relation has been time and again used effectively by many authors for decision making the concept of complete fuzzy relation and incomplete fuzzy relations [8] gives two different approaches for decision making based on the information supplied by the decision maker (DM) [9] in our case the machine. This paper has been organized in the following way section 2 , deals with existing work ,3 with the proposed work , 4 with implementation and section 5 with results and conclusion.

\section{Existing work}

There are numerous face recognition techniques that have been proposed in the last few decades [7]. This existing techniques can be broadly divided into few categories [16]. Due to the simplicity and the efficiency for the feature extraction and representation , many subspace analysis methods, such as principal component analysis PCA, linear discriminant analysis LDA, independent component analysis ICA and locality preserving projections LPP , are widely used for face recognition [1][2]. In 1960, digital image processing was proposed with semi automated systems. In order to locate the major features different marks were made on the photographs. These system uses features like mouth, eyes, and nose. Next to get the common reference points the ratios and distances were calculated and then compared with the database [7]. In 1970's Harmon, Goldstein and Lesk introduced their system [2]. The system had 21 subjective makers having tip thickness and hair color. This was difficult while automating the system because many complex measurements were made by hand. Fisher and Elschlagerb projected a new advancement to face recognition. Another approach is connectionist technique which is used to categorize human faces using two things which are gestures and a set of classifying markers. This technique is normally applied on neural network principles and 2-dimensional pattern recognition. In normal networks a huge training database of faces is needed which required too much 
time to train the whole system to get the desired results. [7] Shariff, Khalid, Raza, Mohosin proposed a mechanized technique for general pattern recognition.

\section{Proposed work}

In the proposed system, we use Gabor filters for face recognition [7] . Gabor wavelets are widely and successfully used in face recognition due to their biological relevance and computational properties. The Gabor wavelets can be defined as follows [16],

$$
\psi_{u, v}(z)=\frac{\left\|k_{u, v}\right\|^{2}}{\sigma^{2}} e^{\frac{-\left\|k_{u, v}\right\|^{2}}{z \sigma^{2}}\left[e^{i\left(k_{u, v}\right)}-e^{\frac{-\sigma^{2}}{z}}\right]}
$$

Where $u$ and $v$ denote the orientation and scale of Gabor kernels, $z=(x, y),\|$.$\| denotes the norm$ operator, and the wave vector $k_{u, v}$ is defined as follows,

$$
k_{u, v}=k_{v} e^{i \varphi_{u}}
$$

Where $k_{v}=\frac{k_{\max }}{f^{v}}, \varphi_{u}=\frac{\pi u}{8} k_{\max }$

Is the maximum frequency and $f$ is the spacing factor between kernels in the frequency domain. The gabor kernels are all self similar since they can be generated from one filter, the mother wavelet by scaling and rotation via the wave vector $k_{u, v}$. Each kernel is a product of a Gaussian envelope and a complex plane wave, while the first term in the square bracket determines the oscillating part of the kernel and the second term compensates for the DC value. In each filtered image we find the maximum intensity points dynamically and are then marked as fiducial points ( Landmarks on faces) [7]. The main objective in the proposed work is to match the face with the faces in the database [5]. For this we use the concept of complete Fuzzy preference relation. The leftmost corner point of each faces which are stored in the data base are marked as the origin and respect to this point the distances of every other fiducial point is calculated by the distance formula. This distance data of each points for every face are then stored in the database. Next in the face which is to be matched, the same coordinate system is used and the distances of each fiducial points are calculated. Now the distance of each fiducial point is compared with the already stored data and a complete fuzzy preference relation matrix is obtained. The largest eigen value of this matrix is obtained and the corresponding eigen vector is determined from which the decision is being made [8]. The step wise rules for the method which we are proposing in this paper can be schematically given by the flowchart as follows.
Flow chart for the proposed method.

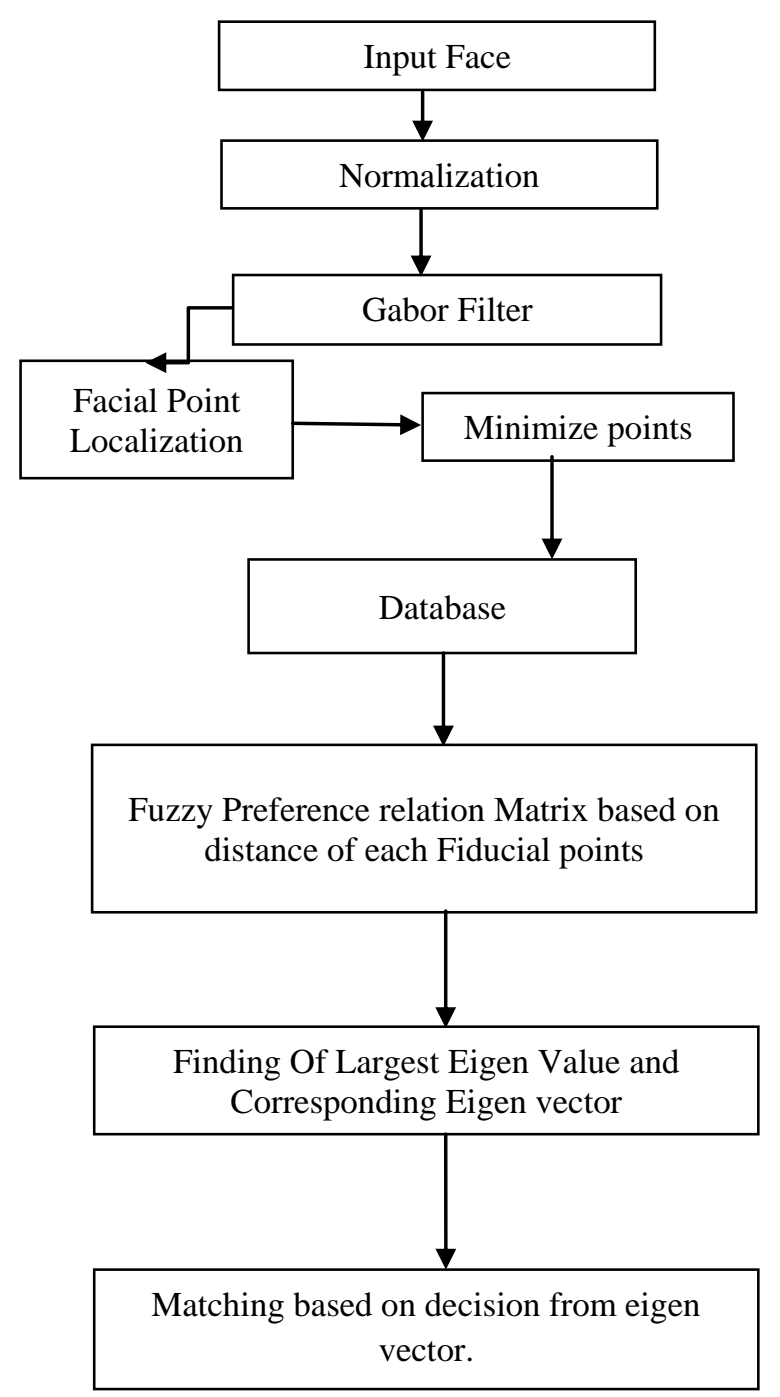

\subsection{Complete fuzzy preference relation}

In the process of decision making [8], a DM generally needs to compare a set of decision alternatives with respect to a criterion and construct a preference relation. Complete fuzzy preference relation is a common preference relation which can be described as follows [9].

Let $X=\left\{x_{1}, x_{2}, x_{3}, \ldots \ldots \ldots x_{n}\right\}$ be a finite set of alternatives. A complete fuzzy preference relation " $P$ " on $X$ is a fuzzy set on the product set " $X \times X$ " that is characterized by a membership function $\mu_{p}: X \times X \rightarrow$ $[0,1]$. The DM (Machine in our case) preferences on $X$ are given by a complete fuzzy preference relation $P=\left(p_{i j}\right)_{n \times n}$, where $p_{i j} \in[0,1] p_{i j}+p_{j i}=1$,

$p_{i i}=0.5 \forall i, j$ and $p_{i j}$ denotes the preference degree or the intensity of the alternative $x_{i}$ over $x_{j}$. In particular, $p_{i j}=0$ indicates that $x_{j}$ is absolutely preferred to $x_{i} ; p_{i j}<0.5$ indicates that $x_{j}$ is preferred 
to $x_{i} \cdot p_{i j}>0.5$ indicates that $x_{i}$ is preferred to $x_{j}$. $p_{i j}=0.5$ indicates indifference between $x_{i}$ and $x_{j}$.

\subsection{Some properties of complete fuzzy preference relation}

1.Tanino (1984) introduced the concept of multiplicative transitivity

$$
\frac{p_{j i} p_{k j}}{p_{i j} p_{j k}}=\frac{p_{k i}}{p_{i k}} \text { where } p_{i j}>0 \forall i, j .
$$

2.Herrera-Viedma et.al. (2004), Xu (2003), and Xu and $\mathrm{Da}$ (2008) rewrote equation 1 . As

$$
p_{i k} p_{k j} p_{j i}=p_{i j} p_{j k} p_{k i} \forall i, j, k
$$

3.Triangle condition (Luce and Suppes (1965), Herrera-Viedma et.al.(2004):

$$
p_{i j}+p_{j k} \geq p_{i k} \forall i, j, k
$$

4.Weak transitivity (Tanino (1984), Herrera-Viedma et.al. (2004):

$$
p_{i j} \geq 0.5, p_{j k} \geq 0.5 \Rightarrow p_{i k} \geq 0.5 \forall i, j, k
$$

5.Max-Min transitivity ( Zimmermann (1991), Herrera-Viedma et.al. (2004)):

$$
p_{i k} \geq \min \left\{p_{i j}, p_{j k}\right\} \forall i, j, k .
$$

6.Max-Max transitivity ( Dubois and Prade (1980), Zimmermann (1991), Herrera-Viedma et.al. (2004)):

$$
p_{i k} \geq \max \left\{p_{i j}, p_{j k}\right\} \forall i, j, k \text {. }
$$

7.Restricted Max-Min transitivity (Tanino (1984), Herrera-Viedma et.al. (2004)):

$$
p_{i j} \geq 0.5, p_{j k} \geq 0.5 \Rightarrow p_{i k} \geq \min \left\{p_{i j}, p_{j k}\right\} \forall i, j, k
$$

8.Restricted Max-Max transitivity (Tanino (1984), Herrera-Viedma et.al. (2004)):

$$
p_{i j} \geq 0.5, p_{j k} \geq 0.5 \Rightarrow p_{i k} \geq \max \left\{p_{i j}, p_{j k}\right\} \forall i, j, k
$$

Saaty (1980) introduced the well known eigen vector method to determine the priority vector " $w$ " of the general multiplicative preference relation : of $A$.

$A w=\lambda_{\max } w$ where $\lambda_{\max }$ is the largest eigen value

Similarly let $w=\left(w_{1}, w_{2}, \ldots \ldots \ldots \ldots w_{n}\right)^{T}$ be the priority vector of the fuzzy preference relation $P=$ $\left(p_{i j}\right)_{n \times n}$ where $w_{i}>0, i=1,2, \ldots . n . \sum_{i=1}^{n} w_{i}=1$. If $P=\left(p_{i j}\right)_{n \times n}$ is a multiplicative consistent complete fuzzy preference relation, then such a preference relation is given by

$$
p_{i j}=\frac{w_{i}}{w_{i}+w_{j}} \forall i, j
$$

In this case $P$ can be expressed as follows

$$
P=\left[\begin{array}{ccccc}
\frac{w_{1}}{w_{1}+w_{1}} & \frac{w_{1}}{w_{1}+w_{2}} & \ldots \ldots \ldots & \frac{w_{1}}{w_{1}+w_{n}} \\
\vdots & \ldots \ldots \ldots \ldots \ldots \ldots & \vdots \\
\frac{w_{n}}{w_{n}+w_{1}} & \frac{w_{n}}{w_{n}+2} & \ldots \ldots \ldots & \frac{w_{n}}{w_{n}+w_{n}}
\end{array}\right]
$$

From equation 2. It follows that the following system of equation can be given

$$
\begin{gathered}
\frac{w_{i}}{w_{i}+w_{1}}\left(w_{i}+w_{1}\right)+\frac{w_{i}}{w_{i}+w_{2}}\left(w_{i}+w_{2}\right) \\
+\cdots \ldots \ldots \ldots \ldots \ldots \ldots \ldots \ldots \ldots \ldots \ldots \\
+\frac{w_{i}}{w_{i}+w_{n}}\left(w_{i}+w_{n}\right)=n w_{i} \\
i=1,2, \ldots \ldots \ldots \ldots n .
\end{gathered}
$$

Using the general complete fuzzy preference relation $P=\left(p_{i j}\right)_{n \times n}$ instead of the multiplicative consistent complete fuzzy preference relation in equation 4 , and using $\lambda_{\max }$ instead of $n$ it follows that ( Lipovetsky and Michael Conklin 2002)

$$
\begin{aligned}
& p_{i 1}\left(w_{i}+w_{1}\right)+p_{i 2}\left(w_{i}+w_{2}\right)+\cdots \ldots . .+p_{i n}\left(w_{i}+\right. \\
& \left.w_{n}\right)=\lambda_{\max } w_{i} \quad \forall i
\end{aligned}
$$

Where $w_{i}>0, i=1,2, \ldots \ldots . n . \quad \sum_{i=1}^{n} w_{i}=1$

By solving equation (3) together with $\sum_{i=1}^{n} w_{i}=1$ we can get the value of $\lambda_{\max }$ and the priority vector $w=\left(w_{1}, w_{2}, \ldots \ldots w_{n}\right)^{T}$ corresponding to $\lambda_{\max }$.

\section{Implementation}

The whole methodology proposed above is implemented on a input face as shown below.

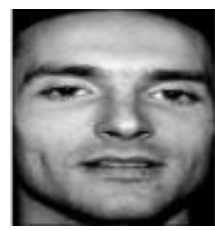

Fig 1: The Input Face

\subsection{Normalisation}

The following are the steps for image normalization which is applied over all the stored images in the database as well as the input face which is to be 
recogonised. The following are the steps of normalization.

1.Rescaling to $128 \times 128$

2.Pixels adjustment:

In this step, image pixel intensities are used, such that the standard deviation of image pixel is one and mean zero.

3.Boundaries are smoothed.

\subsection{Gabor filter}

In this step gabor filter is applied on the input face [7]. The sample 40 gabor filters are shown below in fig (1) [7] . This figures are obtained using five dissimilar scales and eight angles are engaged and in result of it 40 gabor filters are acquired fig (2)[7].

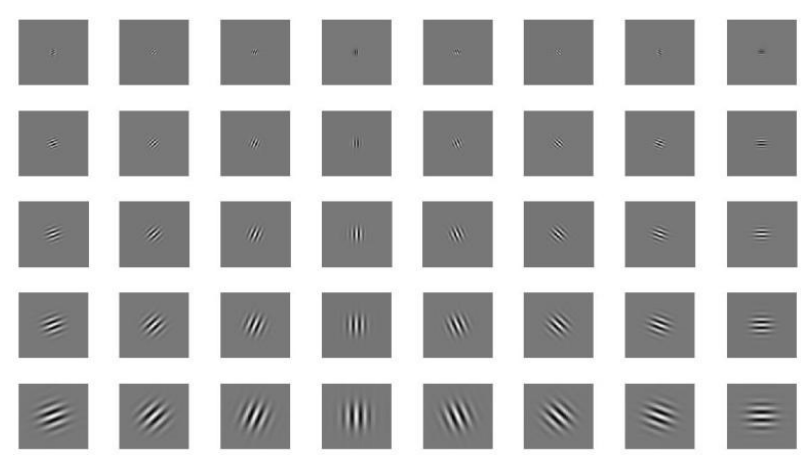

Fig 2: Gabor Filter

\subsection{Applying filter on the image}

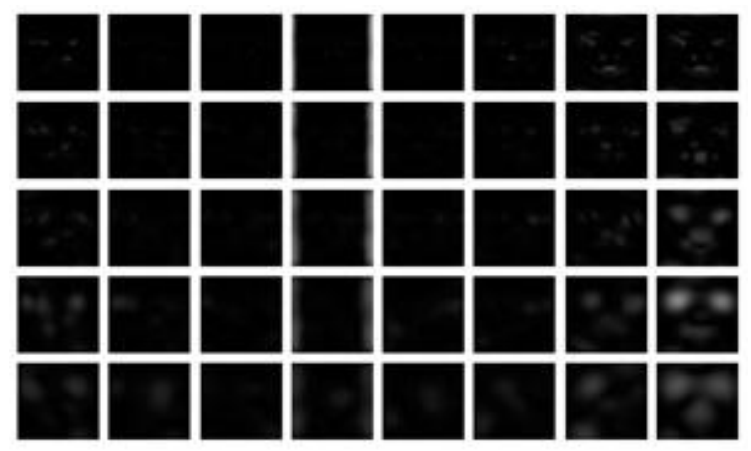

Fig 3: After applying Gabor Filter

Where the input image $I(x, y)$ and the resultant image $J(x, y)$ of gabor filter. When the original image with gabor filter is multiplied. A new image is acquired which is equal to $J(x, y)$, where $x$ and $y$ is the height and width of the image respectively. From [7],

$$
J_{i, j}(x, y)=I(x, y) \times g_{i j}(x, y)
$$

\subsection{Fiducial point localization}

After applying the gabor filter 40 images are required with different angles and orientations. Then the maximum intensity points are found in each image. After that 40 points on image are calculated [7][16] . To find this face points following equations is used

$\sum_{i=1}^{N_{1}} \sum_{j=1}^{N_{2}}\left(\max \left(X_{i j}\right)\right)$ where $X=I_{i, j}$

$$
I=\text { Intensity of coordinate } i, j \text {. }
$$

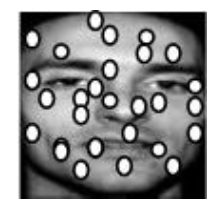

Fig 4: The circles in the face showing intensity points

The maximum intensity points are shown in the figure (4) above.

$N_{1}$ and $N_{2}$ are the width and height of image. By using this equation one point is found on each image out of 40 gabor filtered images. Then the distance is calculated to minimize the points. The minimum distance possible between two points is defined to maximize the number of points.

The reduced number of points are shown below in the figure (5).

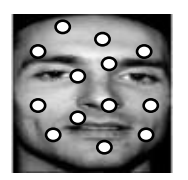

Fig 5: The reduced number of points are marked

\subsection{Distance matrix for fiducial point in terms of complete fuzzy preference relation}

To obtain the distance matrix for fiducial point in terms of complete fuzzy preference relation we take six fiducial points for the person whose face is being detected and we also keep the data of six fiducial points for six different persons in the database of the system. We use the matrix given in [8].

Faces in Database

$$
\mathrm{A}=\text { Input Face }\left[\begin{array}{cccccc}
0.5 & 0.3 & 0.6 & 0.3 & 0.8 & 0.3 \\
0.7 & 0.5 & 0.7 & 0.6 & 0.6 & 0.8 \\
0.4 & 0.3 & 0.5 & 0.4 & 0.5 & 0.7 \\
0.7 & 0.4 & 0.6 & 0.5 & 0.4 & 0.8 \\
0.7 & 0.2 & 0.3 & 0.2 & 0.3 & 0.5
\end{array}\right]
$$

By using equation (3) above and $\sum_{i=1}^{6} w_{i}=1$, we can establish the following system of equations.

$$
\begin{aligned}
3.28 w_{1}+0.3 w_{2} & +0.58 w_{3}+0.3 w_{4}+0.8 w_{5}+0.3 w_{6} \\
& =\lambda_{\max } w_{1}
\end{aligned}
$$




$$
\begin{aligned}
0.7 w_{1}+4.38 w_{2} & +0.7 w_{3}+0.6 w_{4}+0.6 w_{5}+0.8 w_{6} \\
& =\lambda_{\max } w_{2} \\
0.42 w_{1}+0.3 w_{2} & +3.34 w_{3}+0.4 w_{4}+0.5 w_{5}+0.7 w_{6} \\
& =\lambda_{\max } w_{3} \\
0.7 w_{1}+0.4 w_{2}+ & 0.6 w_{3}+3.899 w_{4}+0.4 w_{5}+0.8 w_{6} \\
= & \lambda_{\max } w_{4} \\
0.2 w_{1}+0.4 w_{2}+ & 0.5 w_{3}+0.6 w_{4}+3.405 w_{5}+0.7 w_{6} \\
= & \lambda_{\max } w_{5} \\
0.7 w_{1}+0.2 w_{2}+ & 0.3 w_{3}+0.2 w_{4}+0.3 w_{5}+2.699 w_{6} \\
= & \lambda_{\max } w_{6}
\end{aligned}
$$

This system of equations can be transformed into the following eigenvalue problem

$$
B w=\lambda_{\max } w
$$

Together with $\sum_{i=1}^{6} w_{i}=1$, where,

$$
B=\left[\begin{array}{llllll}
3.9 & 0.3 & 0.6 & 0.3 & 0.8 & 0.3 \\
0.7 & 4.9 & 0.7 & 0.6 & 0.6 & 0.8 \\
0.4 & 0.3 & 3.3 & 0.4 & 0.5 & 0.7 \\
0.7 & 0.4 & 0.6 & 3.9 & 0.4 & 0.8 \\
0.2 & 0.4 & 0.5 & 0.6 & 3.4 & 0.7 \\
0.7 & 0.2 & 0.3 & 0.2 & 0.3 & 2.7 \\
& & & & &
\end{array}\right]
$$

By solving this eigenvalue problem we get the value of $\lambda_{\max }$ and the priority vector " $w$ " corresponding to $\lambda_{\max }$ as follows,

$$
\lambda_{\max }=6.00
$$

$$
\text { and } w=(0.137,0.289,0.136,0.201,0.151,0.086)^{T}
$$

Ranking of the alternatives based on priority vector $" w$ " can be done as follows,

$$
w_{6}<w_{3}<w_{1}<w_{5}<w_{4}<w_{2}
$$

and thus the most preferable alternative is $w_{2}$.

Therefore from the above drawn conclusion we can say the input face resembles the face of person stored as $M_{2}$ in the database of the system.

To find the largest eigen value and the corresponding priority vector we will apply the numerical method if the order of the matrix becomes large for which we suggest a algorithm which we have used for $\mathrm{C}$ programming to obtain the largest eigen value.

\subsection{Algorithm to determine the largest eigen value}

Step1. Start the program
Step2. Read $n$ and the given matrix $\left(a_{i j}\right)$ for $i=$ 1(1) $n J=1(1) n$;

Step3: For $i=1(1) n$, set $x_{i}=1$

Step4: For $k=1(1) 20$

Step5: Fori $=1(1) n$

Step6: Set $y_{i}=0$

Step7: For $j=1(1) n$

Step8: set $y_{i}=y_{i}+a_{i j} x_{j}$

Step9: Next $j$, Next $i$

\begin{tabular}{|c|c|c|c|c|}
\hline & $\begin{array}{c}\text { Total } \\
\text { Images }\end{array}$ & Matched & $\begin{array}{c}\text { Unmatche } \\
\text { d }\end{array}$ & $\begin{array}{l}\text { Result } \\
\text { in \% }\end{array}$ \\
\hline \multirow{7}{*}{$\begin{array}{l}\text { Peoposed } \\
\text { Techniques }\end{array}$} & 2 & 2 & 0 & 100 \\
\hline & 10 & 10 & 0 & 100 \\
\hline & 14 & 13 & 1 & 92.85 \\
\hline & 20 & 18 & 2 & 90.00 \\
\hline & 25 & 23 & 2 & 92.00 \\
\hline & 30 & 28 & 2 & 93.3 \\
\hline & 35 & 32 & 3 & 91.4 \\
\hline \multirow{3}{*}{$\begin{array}{c}\text { Existing } \\
\text { Techniques }\end{array}$} & \multicolumn{3}{|c|}{ Eigenface [12] } & 80.00 \\
\hline & \multicolumn{3}{|c|}{ Elastic graph matching [13] } & 80.00 \\
\hline & \multicolumn{3}{|c|}{ EBGM [14] } & 75.29 \\
\hline
\end{tabular}

Step10: $m=y_{1}$

Step11: For $i=2(1) n$

Step12: If $m<y_{i}$, set $m=y_{i}$

Step13: Next $i$

Step14: For $i=1(1) n$ set $x_{i}=\frac{y_{i}}{m}$

Step15: Write the numerically largest eigen value.

Step16. Stop.

\section{Experiments and results}

To measure he effectiveness and accuracy of the proposed method the standard database 94 [15][7] is used. For this twenty person's images were selected. There were twenty images of each person in the database.

Comparison of Result:-

In the proposed method total 136 images were compared of which 10 were not matched. So overall percentage of success in this method is $92.64 \%$. 


\section{Concluding Remarks}

The proposed technique of face recognition gives a new approach based on complete fuzzy preference relation. The use of complete fuzzy preference relation in decision making while recognizing the faces is better than many other method. The reason being the fact that a human face has the possibility of transformation it may become fat or thin depending upon the physical changes of human body, in this extreme cases also this method is expected to give better results, The efficiency of the method can be further enhanced by increasing the number of fiducial points. However the efficiency of the method largely depends on the accuracy in construction of the fuzzy preference matrix. This technique also opens up a new approach in face recognition techniques for future research.

\section{References}

[1] M.Turk and A.Pentland “ Eigenfaces for recognition” journal of cognitive science, 1991, pp 71-86.

[2] A.J. Goldstein, L.D. Harman, and A.B. Lesk, "Identification of human faces" Proc. IEEE, May 1971, Vol 59, no. 5, 748-760.

[3] W.Zhao,R.Chellappa,P.J.Phillips, and A.Rosenfield. "Face recognition: a litertature surveu. ACM computing surveys" 35(4), 2003.pp. 399-458.

[4] K.karibasappa, K.S.Patanaik, A.Konar, A.K.Mondal (1999). "Image matching with fuzzy correlation descriptions", Proc. Int.Con. on "Information Technology" (CIT 99) held in Bhubaneswar pp 121-125.

[5] L.Chaddah,et.al. recognition of human face using interconnection network. J.IETE, 42 (425), (1996), 261-267.

[6] L.Wiskott, et.al.. "Face recognition by elastic bunch graph matching". IEEE Trans. PAMI, 19(7), (1997), 725-779.

[7] M.Sharif, A.Khalid,M.Raza, S.Mohasin.”face recognition using gabor filters" journal Of Applied computer science and Mathematics. 11(5),2011,pp53-57.

[8] Z.Xu. "A procedure for decision making based on Incomplete fuzzy preference relation". Fuzzy optimization and decision making, 4-2005, pp 175189.

[9] Z.S. Xu and Q.L. Da " An approach to improving consistency of fuzzy preference Matrix" . Fuzzy optimization and decision making 2003 ,2, 3-12.

[10] T.L. Satty. The analytical hierarchy process. New York: McGraw-Hill
[11] T.S. lee “ Image representation using 2D Gabor wavelets”, IEEE Trans. PAMI, Vol18, no.10,1996. pp 959-971.

[12] R.Baron, “ Mechanisms of Human facial recognition” Int.J.Man-Machine studies, vol 15, 1981 ,pp 137-178.

[13] Chow and Li, “ Towards a system for automatic facial feature detection", Pattern recognition, vol.26, No. 12, 1993 ,pp 1739-1755.

[14] L.Shen, L.bai, “ Mutual Boast learning for selecting gabor features for face recognition", Pattern recognition Letters, Vol.27, No. 15,2006, pp. 1758-1767.

[15] V.Bruce,"Recognizingfaces.”London:Erlabam,198 8.

[16] L.Pratibhan, "A novel face recognition algorithm with support vector machine classifier" IJMSC ,Vol.1, No.1, 2011, pp 28-34.

S.Biswas is an Asstistant Professor in the Department of Mathematics, NIT, Jamshedpur, INDIA. Major research interest includes Fuzzy Mathematics.

Dr.S.Jha is an Asstistant Professor in the Department of Mathematics, NIT, Jamshedpur, INDIA. Major research interest includes Fuzzy Mathematics, Approximation Theory.

Dr.R.Singh is an Asstistant Professor in the Department of Mathematics, NIT, Jamshedpur, INDIA. Major research interest includes Fuzzy Mathematics, Special functions, Numerical analysis. 